\title{
Pulse oximetry in the accident and emergency department
}

\author{
C. J. HOLBURN \& M. J. ALLEN \\ Accident and Emergency Department, Leicester Royal Infirmary, Leicester, England
}

\section{SUMMARY}

The early detection and treatment of hypoxia is important to reduce patient morbidity in the accident and emergency department. At present, the commonly used methods all have practical difficulties in the urgent situations that prevail in the department. Pulse oximetry has recently become a method of choice in many anaesthetic and intensive care units for the continuous monitoring of oxygenation and the early detection of hypoxia. As similar conditions prevail in the accident and emergency department, we have attempted to evaluate its ease of use and the quality of information obtained in our department.

Patients presenting with chest pain to an accident and emergency department have their oxygen saturation measured by the pulse oximeter finger probe prior to the commencement of oxygen therapy. After $5 \mathrm{~min}$ of oxygen therapy, the measurement was repeated. Our result showed that although no patients could be judged as hypoxic on clinical grounds the pulse oximeter showed, within $30 \mathrm{~s}$ of admission, that a number had an abnormal oxygen saturation. Continuous monitoring with the probe after the application of low flow oxygen therapy also aided in monitoring their treatment and this improvement was again easily and quickly recorded.

Our experience shows that the pulse oximeter may be a useful tool for evaluating hypoxia and oxygen therapy in the accident and emergency department and we feel that we should be one of the groups who should reply in the positive to Zorab's question, 'Who needs pulse oximetry?' (Zorab, 1988).

\section{INTRODUCTION}

Cellular hypoxia precedes cell death in a large number of insults to the body.

In the accident and emergency department, changes in the patient's oxygenation must be rapidly assessed and corrected to prevent unnecessary morbidity and mortality.

Correspondence: Mr C. F. Holburn, Senior Registrar, Accident and Emergency Department, Dudley Road Hospital, Dudley Road, Birmingham, England. 
The clinical detection of hypoxia uses the presence or absence of cyanosis as a guide $\frac{\mathrm{m}}{3}$ but this is unreliable (Comroe et al., 1947) especially in the artificial lighting of many $\frac{\mathbb{Q}}{6}$

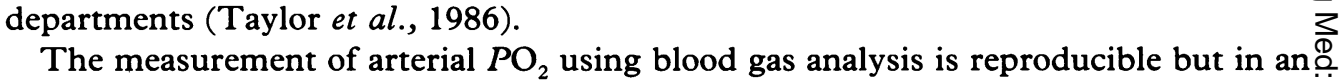
unstable patient requires multiple invasive procedures giving intermittent values (Lam, $\underset{\vec{s}}{\stackrel{9}{9}}$ 1987).

Transcutaneous oxygen monitoring does provide continuous information but the $\frac{\bar{C}}{0}$ sensor requires a stabilization period of about $30 \mathrm{~min}$ before reading can be commenced. $\frac{\bar{\omega}}{\bar{D}}$ As the sensor also requires the skin to be heated to $41^{\circ} \mathrm{C}$ to arterialize the capilliary bed, $\stackrel{\varnothing}{\varrho}$ burns of the sensor area have been reported (Yelderman et al., 1983).

Measurement of oxygen saturation by detecting the difference in absorption of light $\vec{\circ}$ by haemoglobin and oxy-haemoglobin had been developed for in vitro analysis of blood $\overrightarrow{-}$ and a modification of this equipment using 8 wavelengths of light became the Hewlett Packard ear oximeter. While this equipment could give accurate and continuous $\frac{\mathbb{D}}{3}$ information it was bulky, expensive and unsuitable for routine clinical use (Knill et al. ف) 1982).

With the advent of light emitting diodes (LEDs) the bulk of the detection system was $\vec{\omega}$ reduced, but this unheated and simpler equipment measured the total absorbance fromo tissues, veins and capillaries in addition to the absorbance due to arterial oxygensaturation.

The pulse oximeter analyses not only the absorbance of light but by using microprocessors the changes of the signal produced by arterial pulsations. By excluding the $\vec{\oplus}$ absorbance of the constant part of the signal the arterial saturation is recorded and hgीs. been shown to reflect simultaneous invasive measurements accurately (Yelderman et a⿳亠丷厂犬, 1983; Mackenzie, 1985).

The modern pulse oximeter is a compact and easily mobile piece of equipmento (Figure 1). It has been shown to be accurate over a wide variety of temperatures, cardiac index and mean arterial pressure. If, however, the equipment cannot identify a pulse $\overrightarrow{\vec{O}}$

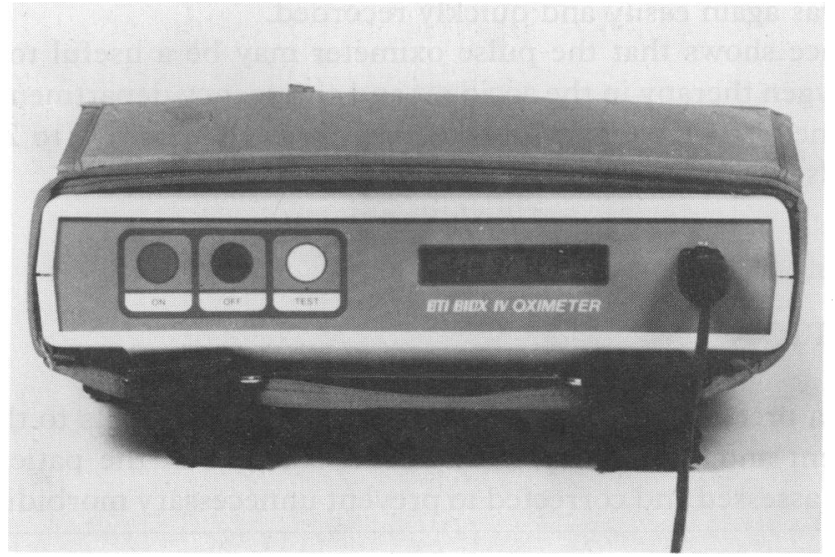

Fig. 1 The Biox 4 pulse oximeter showing oxygen saturation $\left(\mathrm{SaO}_{2}\right)$ and the pulse rate $(\mathrm{BPM})$. 
due to low perfusion of the vascular bed it displays a 'low perfusion' message and not an inaccurate value.

This instrument has now been introduced into a wide variety of clinical areas most notably the anaesthetic room and intensive care unit. A recent leader in the British Medical fournal posed the question, 'Who needs pulse oximetry?' (Zorab, 1988) but did not specifically mention the accident and emergency department. No reports of its use have been found in the British accident and emergency literature, and we therefore undertook a study to assess the potential of the pulse oximeter in the accident and emergency department.

\section{METHOD}

Patients presenting to the accident and emergency department with the complaint of 'chest pain' who required monitoring were included in the study.

The BTI Biox 4 Oximeter was used in the study. This displays the oxygen saturation and the patient's pulse rate (Figure 1).

Directly on admission to the accident and emergency department each patient had the finger probe placed on the index finger (Figure 2) while breathing room air. The machine was allowed to settle to a constant oxygen saturation figure and this was recorded if the pulse rate reading was within $5 \mathrm{bpm}$ of the radial pulse rate.

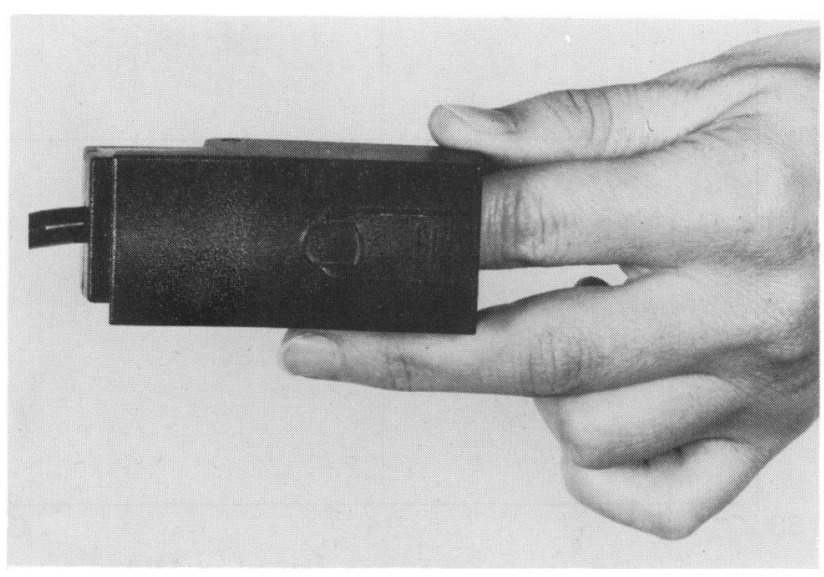

Fig. 2 The pulse oximetry finger probe in position.

Immediately following this reading the patient was then given controlled oxygen therapy, $4 \mathrm{l} / \mathrm{min}$ by Hudson Mask, an approximate $\mathrm{FiO}_{2}$ of $35 \%$, this being the recommended treatment for chest pain of cardiac origin (Yates \& Redmond, 1986).

After 5 min of oxygen the oxygen saturation displayed on the machine was recorded. 


\section{RESULTS}

Fifty patients ( 32 male, 18 female) average age 59 years (range 23-84) were studied. $\frac{3}{8}$ None had clinical evidence of cyanosis or respiratory distress in the accident and $\stackrel{?}{?}$ emergency department. All had stable cardiovascular systems, none were hypotensive $\stackrel{\vec{\rho}}{\stackrel{\rho}{\rho}}$ and none had evidence of bleeding.

The initial pulse oximetry reading varied between $88-100 \%$ saturation (normal $>94 \%)(\mathrm{Oh}, 1985)$ and this was recorded within $1 \mathrm{~min}$ of the patient entering the department.

After $5 \mathrm{~min}$ of oxygen therapy the second reading was obtained and as expected is showed a higher value (Figure 3 ) with all values being within the normal range. All $\overrightarrow{0}$ those with abnormal initial values were admitted to hospital most $(12 / 13)$ with chest pain of cardiac origin.

None of the patients with abnormal initial saturations could be identified by clinical $\stackrel{\oplus}{3}$ means and indeed some were felt by the nursing staff not to require oxygen therapy.

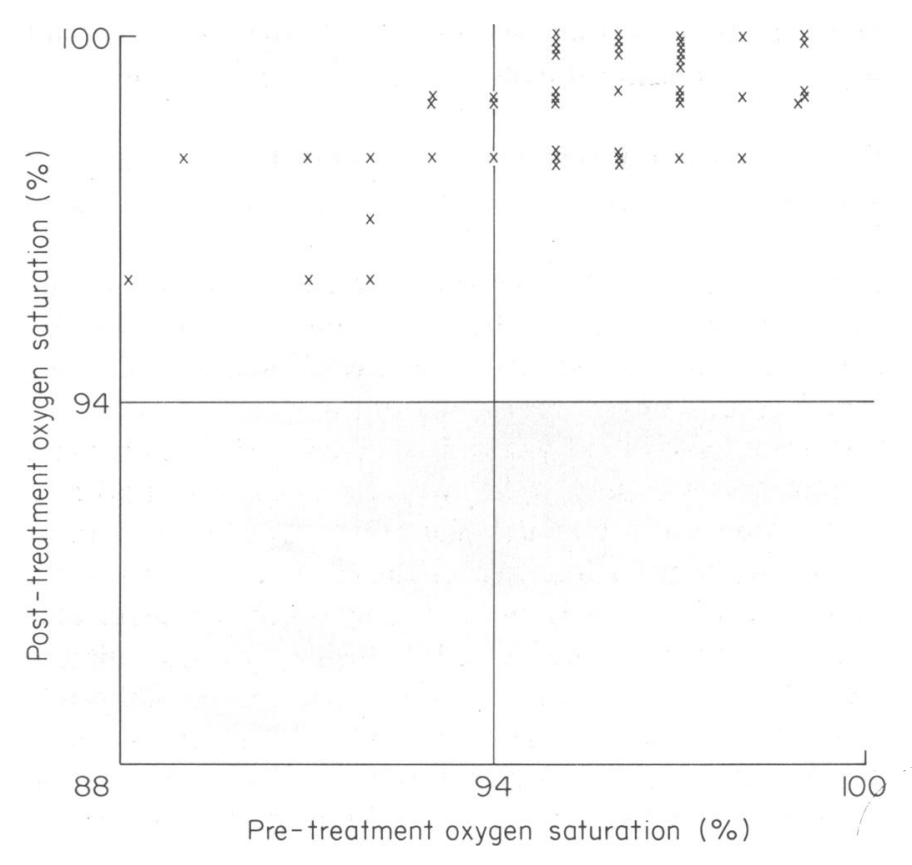

Fig. 3 The pre- and post-oxygen therapy saturation. The lines show the lower limit of normal. 


\section{DISCUSSION}

Unrecognized and uncorrected hypoxaemia can increase the morbidity associated with a large number of the conditions that present to the accident and emergency department. Many of the therapeutic interventions that are used in the acutely ill aim to improve the level of tissue oxygenation and it is important that the results of these interventions can be quickly assessed.

The clinical detection of hypoxia is difficult (Comroe et al., 1947) and is affected by a wide variety of environmental conditions (Taylor et al., 1986). The rapidity with which a patient's oxygenation can change has been recorded in the anaesthetic literature and the pulse oximeter has been instrumental in alerting anaesthetists to the hypoxaemia even minor insults can cause (Mihn et al., 1985; Tyler et al., 1985).

The same rapidly changing conditions exist in the accident and emergency department and a method of evaluating a patient's oxygenation which is reliable and responds quickly to changing conditions is needed.

We have attempted to use the pulse oximeter for this purpose and have found it to be easy to use and in the group of patients studied gave valuable information both before and during oxygen therapy.

It alerted us to the fact that clinical judgement of oxygenation is difficult and that within a group of patients presenting with 'chest pain' some may have deficient oxygen delivery.

Although the total numbers in this sample are small, all of the patients with saturation values below $94 \%$ were admitted to hospital while some with higher values were discharged.

We cannot determine whether the low saturation obtained on admission was as a result of pre-existing disease or due to the presenting condition but in patients with myocardial ischaemia any reduction in saturation from normal imposes a greater load on the heart which can further damage the myocardium.

As the patients did not have blood gas analysis during this study we cannot be sure that the initial value represents just oxyhaemoglobin as other abnormal haemoglobin types can give a false value with this particular equipment but we can be sure that the increased value does represent an increase in the saturation. As the basic method of evaluation of oxygen saturation by this method relies on the presence of a pulse wave at the recording point the technique as described in this paper is not suitable for use in the hypotensive patient. This may preclude its use in trauma although we have no experience of its use in this field. As the technology of the instruments improves, the detection of oxygen saturation at lower blood pressures may become easier.

The recent increase in litigation has prompted editorial comment (Mathias et al., 1987) on the need for early detection of iatrogenic complications of treatment. The author recommends the pulse oximeter as a useful tool for the anaesthetist. In accident and emergency departments the same vigilance is necessary and the pulse oximeter provides the information quickly and in an easily readable form.

As the cost of investigations increases the use of reusable non-invasive techniques to reduce the number and frequency of blood gas analysis without replacing them completely will become an important consideration in clinical practice (Dautzenberg, 1986). 


\section{CONCLUSIONS}

In a small pilot study oxygen saturation was quickly and easily measured by the non- $\frac{2}{8}$ invasive pulse oximeter in a group of acutely ill patients presenting to an accident and emergency department.

Further studies are required to establish the exact role of this instrument in the normal day-to-day practice of emergency medicine.

\section{REFERENCES}

Comroe J. H. \& Botalho S. (1947) The unreliability of cyanosis in the recognition of arterial anoxemia. American fournal of the Medical Sciences 214, 1-6.

Dautzenberg B., Gallinari C., Moreau A. \& Sors C. (1986) The advantages of real-time oximetry over intermittent arterial blood gas analysis in a chest department In Pulse Oximetry, Payne J. P. \& Severinghaus J. W.(eds), pp. 63-5. Springer-Verlag, Berlin.

Knill R. L., Clement J. L., Kieraszewicz et al., (1982) Assessment of two noninavasive monitors of arteria $\vec{\omega}$ oxygenation in anaesthetized man. Anaesthesia $\mathbb{E}$ Analgesia 61, 582-6.

Lam A. M. (1987) Continuous arterial $\mathrm{PO}_{2}$ monitoring, In: Monitoring of Oxygen: A Panel Summary.

Canadian Anaesthetists' Society fournal 34, 56-63.
Mackenzie N. (1985) Comparison of a pulse oximeter with an ear oximeter and an in-vitro oximeter. fournal of Clinical Monitoring 1, 156-60.

Mathias J. A. \& Lunn J. N. (1987) Minimal monitoring and vigilance. Anaesthesia 42, 683-4.

Mihn F. G. \& Halperin B. D. (1985) Noninvasive detection of profound arterial desaturations using a pulse oximetry device. Anaesthesiology 62, 85-7.

Oh T. E. (ed) (1985) Intensive Care Manual. Butterworth, London.

Taylor M. B. \& Whitwam J. G. (1986) The current status of pulse oximetry. Anaesthesia 41, 943-9.

Tyler I. L., Tantisira B., Winter P. M., et al., (1985) Continuous monitoring of arterial oxygen saturation with pulse oximetry during transfer to the recovery room. Anaesthesia $\mathcal{E}$ Analgesia 64 1108-12.

Yates D. W. \& Redmond A. D. (1986) Lecture Notes on Accident and Emergency Medicine. Blackwell Scientific Publications, Oxford.

Yelderman M. \& New W. (1983) Evaluation of pulse oximetry. Anaesthesiology 59, 349-52.

Zorab J. S. M. (1988) Who needs pulse oximetry? British Medical fournal 296, 658-9. 